\title{
Towards an Optimal Information Architecture Model for Mobile Multimedia Devices
}

\author{
Timo-Pekka Viljamaa ${ }^{1}$, Tuomas Vaittinen ${ }^{1}$, and Akseli Anttila ${ }^{2}$ \\ ${ }^{1}$ Nokia Research Center, P.O. Box 407, 00045 Nokia Group, Finland \\ ${ }^{2}$ Nokia Corporation, P.O. Box 407, 00045 Nokia Group, Finland \\ \{Timo-Pekka.Viljamaa, Tuomas.Vaittinen, \\ Akseli.Anttila\}@Nokia.com
}

\begin{abstract}
In this paper we propose an optimal information architecture model for mobile multimedia devices. In our study we first reviewed the information architecture models of four currently popular mobile multimedia devices, Apple iPod Video, Nokia N80, Sony Ericsson W800i and Sony PlayStation Portable (PSP). Then we used Open card sorting method to find out what kind of mental models does people have for optimal information architecture model. Finally we compared these two study results together and created a design proposal.
\end{abstract}

Keywords: information architecture model, card sorting, cluster analysis, mobile multimedia device, mobile multimedia, user-centered design.

\section{Introduction and Objective}

Mobile multimedia devices, such as Apple iPods and Nokia Nseries multimedia phones, are becoming increasingly versatile convergence devices. The user can access a wide variety of different data entities, such as messages, calendar, music, photos, and even TV channels. Designer of such devices is faced with a problem of finding a user-acceptable model to combine the different features and data elements into one UI.

Managing digital documents in computers has been studied by many researchers in the past, and various solutions have been proposed [3]. Studies into document management in mobile multimedia devices however are few in number.

In the face of this increasing complexity and various implemented solutions we decided to conduct a study consisting from background and card sorting studies to determine the user expectations for data organization in mobile multimedia devices. Our main research interest was in finding out an information architecture model with access to all the possible types of data a device can hold from personal information items to various forms of multimedia. We also wanted to compare this model to the existing information architecture models of currently popular mobile multimedia devices.

First we conducted a background study, where four currently popular mobile convergence devices were reviewed to find out how different personal information and multimedia items are categorized in these devices. 
Next we used Open card sorting method to find out the Finnish and US participants' mental models for organizing and categorizing data. The cards contained both personal information and multimedia items, which participants were asked to sort and categorize in the most optimal way they could without any restrictions.

Finally we performed a cluster analysis on the card sorting data, compared the found information architecture to the background study findings, and also created a design proposal for optimal information architecture.

\section{Background Study}

Four currently popular mobile multimedia devices, Apple iPod Video, Nokia N80, Sony Ericsson W800i and Sony PSP, were selected to the background study. Each of the devices has a different main multimedia focus, but as an addition to that all of them offer pretty similar support for other multimedia features and personal information items. The information architecture model of each device for multimedia and personal information items was reviewed and the found main categories were also visualized as shown in Fig. 1. The devices had more functionality, but in this study we were only interested in items from these two categories.

\begin{tabular}{|c|c|}
\hline $\begin{array}{l}\text { Apple iPod Video ( } 5^{\text {th }} \text { Generation) } \\
\text { - Music } \\
\text { - Playlists, Artists, Albums, Songs, Podcasts, Genres, } \\
\quad \text { Composers, Audiobooks } \\
\text { - Photos } \\
\text { - Photo libr ary, Photo album 1,..., Photo album N } \\
\text { - Video } \\
\text { - Video playlists, Movies, Music videos, Video podcasts } \\
\text { - Extras } \\
\text { - Games, Calendar, Notes }\end{array}$ & $\begin{array}{l}\text { Nokia N80 } \\
\text { - Music player } \\
\text { - All tracks, Artists, Albums, Playlists, Genres, Composers } \\
\text { - Gallery } \\
\text { - Images \& video, Tracks, Sound clips, Streaming links, } \\
\text { All files } \\
\text { - My own } \\
\text { - Games, FM radio } \\
\text { - Office } \\
\text { - Notes } \\
\text { - Calendar } \\
\text { - Messaging }\end{array}$ \\
\hline $\begin{array}{l}\text { Sony Ericsson W800i } \\
\text { - File manager } \\
\text { - Pictures, Videos, Sounds, Games } \\
\text { - Walkman } \\
\text { - Artists, Tracks, Playlists, Videos } \\
\text { - Entertainment } \\
\text { - Games } \\
\text { - FM radio } \\
\text { - Organizer } \\
\text { - Calendar, Notes } \\
\text { - Messaging }\end{array}$ & $\begin{array}{l}\text { Sony PlayStation Portable (PSP) } \\
\text { - Photo } \\
\text { - Photo album 1,..., Photo album N } \\
\text { - Music } \\
\text { - Music album 1, ..., Music album N } \\
\text { - Video } \\
\text { - Game }\end{array}$ \\
\hline
\end{tabular}

Fig. 1. Information architecture models of all reviewed devices

\subsection{Background Study Results}

The review of different devices showed that the prevalent method was to categorize the different multimedia and personal information items according to the personal 
information and media typology. The biggest differences were found in the subcategories under the quite similar main categories of each device.

Similarities were found especially in music where iPod's "Music" category and N80's "Music player" category had almost identical subcategories, namely "Playlists/Track lists", "Artists", "Albums", "Songs/All tracks", "Genres" and "Composers". As addition to this, iPod also had subcategories "Podcasts" and "Audiobooks". N80 on the other hand provided access to music also through "Gallery" category, but when accessed this way only songs and albums were shown without any sophisticated subcategories, like in "Music player" category. Similarly W800i provided access to music through two different top-level categories, "File manager" and "Walkman". Through "File manager" category the user could access "Sounds" subcategory containing both songs and folders, while "Walkman" category had similar, but fewer predefined subcategories as iPod and N80, namely "Artists", "Tracks" and "Playlists". PSP's "Music" category on the other hand only contained songs and folders without any predefined subcategories.

In all devices, "Photo" category contained both photos and photo albums. N80 was the only device, where photos and videos were stored under the same category, while in all other devices images and videos were separated to own categories. iPod had most subcategories for "Video" category, namely "Video playlists", "Movies", "Music videos" and "Video podcasts", while in other devices there wasn't any ready made subcategories for videos. Also, none of the reviewed devices made any distinction between the user's self-created videos and public/commercial videos. In W800i videos were accessible through two same categories as in music, "File manager" and "Walkman".

Only N80 and W800i contained FM radio, which in N80 was included to "My own" category together with "Games" subcategory, while in W800i it was placed on the top-level main category. iPod had "Games" subcategory under "Extras" category and PSP had own main category for games. In W800i games could be found from two separate categories, either through "File manager" or "Entertainment".

PSP was the only device that did not contain any personal information items, while all other devices contained at least calendar events and notes, N80 and W800i also messages. In iPod both calendar events and notes were stored as subcategories inside "Extras" category. N80 on the other hand had "Calendar" category for calendar events and notes stored under "Office" category, while in W800i calendar events and notes could be found under "Organizer" category. Both of them contained top-level "Messaging" category.

\section{Card Sorting}

\subsection{Card Sorting Method}

The card sorting method has been well established as a tool to find out the user's mental models for organizing and categorizing data $[6,10]$. The method has been successfully adopted and used in different domains, such as World Wide Web design 
[7, 9], decision support tools for the neonatal intensive care environment [2], requirements engineering [5] or even in sorting different wines to groups [4].

There are two different kinds of card sorting methods, Open and Closed, which can be used to understand how users categorize information in a given domain. Open card sorting method can be used for exploratory analysis of a domain, and for the generation of candidate structures, while Closed card sorting method can be used to comparatively test the suitability of several proposed structures and choose the most suitable one [8].

In our study, we chose to use Open card sorting method, because we wanted to see, what kind of categories people would create for multimedia and personal information items if there were not any predefined categories or restrictions. We were also interested to see how does the card sorting method work with applications and items stored in mobile multimedia devices.

\subsection{Cluster Analysis}

The model produced by participant in a card sorting exercise can be inspected visually and it will provide some understanding of users' mental models. Cluster analysis yields a richer understanding of the data through dendograms (hierarchical structures based on the user's cognitive representations of the domain). We decided to use IBM's EZSort analysis tool to perform cluster analysis on the data and display the results as a dendogram describing the strength of the perceived relationships between pairs of cards [1]. In addition to the quantitative material produced by the cluster analysis, we also conducted pre- and post-test interviews with participants to find richer data about their patterns of convergence device use, and the rationale behind their adopted organizational model.

\subsection{Participants}

The card sorting test was conducted in Helsinki, Finland and in Mountain View, US with total 27 participants. In Finland 18 people ( 6 female, 12 male) participated to the test, and in US we had 9 participants ( 2 female, 7 male). The main recruitment criteria were daily usage of media on the go, such as using a portable music player or a multimedia phone. Most of participants had a background in information technology or in arts and design, and had also different ethnic backgrounds. Age distribution was 24-43 (Finland 24-39, US 30-43) and mean 32 (Finland 29, US 38.5).

\subsection{Cards}

Before the test we created a set of 29 cards listing the data entities a user can access in a convergence device. These entities were known to be already available in high-end mobile phones and other mobile multimedia devices, and included both public and personal media, online and local content, communication and calendar data as shown in Table 1. The cards were then individually printed out in standard playing card size and enclosed in commercial protective plastic sheaths. 
Table 1. Card sorting cards

\begin{tabular}{ll}
\hline Title & Description \\
\hline Audio book & An audio book you saved \\
Audio note & Dictaphone recording you have made \\
Audio stream & Online service \\
Calendar entry & Time and place of event or meeting \\
E-mail & A message you received \\
MMS & Multimedia message you received and saved \\
Network folder & Online folder for sharing things \\
Newspaper & Online articles \\
Note & Text note you have created \\
Picture & Downloaded picture \\
Picture note & Photo taken as a reminder \\
Photo & You took with the phone camera \\
Podcast & Audio recording downloaded from the net \\
Radio channel & Tuning settings for a radio station \\
Radio reminder & Time and channel for a favorite radio program \\
Received image & A picture you got \\
Received video & A video clip someone sent you \\
Recorded music & You recorded with the phone \\
Ring tone & A tone you downloaded \\
Song & A music file (mp3) \\
Text message & You received and saved \\
TV channel & Tuning settings for a mobile TV station \\
TV recording & A recorded TV program \\
TV reminder & Time and channel for favorite program \\
URL & A web link you saved \\
Video clip & Downloaded video \\
Video recording & You made with the phone camera \\
Video stream & Online streaming service \\
Web notifier & A notification of change on a page \\
\hline
\end{tabular}

\subsection{Test Set-Up}

The test was conducted individually for each participant. Two facilitators were present, one taking notes and the other interacting with participant. The test was videotaped. Before the card sorting exercise, participants were asked about their current media usage in a semi-structured interview. The actual card sorting exercise was divided into three stages:

- First the shuffled deck of cards was given to participant who was asked to sort and cluster them according to any model that is logical in his/her mind. Participant was left in peace to sort the cards, but he or she was instructed to ask questions at any time.

- After participant had sorted the cards, he or she was asked to give a descriptive name to each cluster.

- Finally, participant was asked to describe why he or she decided to use this particular sorting strategy, and to elaborate on each cluster. 


\subsection{Card Sorting Results}

In the interview, when participants were asked to describe their clusters, several different grouping schemes came up:

- Media type (e.g. text, radio, video, audio, images)

- Devices that participant associated the media (e.g. iPod, computer, phone)

- Origin (e.g. self-created, received from others, commercial)

- Content location (e.g. local, online, broadcasted)

- Time span (e.g. stuff that needs to be taking care of, things that come up any moment, things that wants to spend time on)

Typically, participants did not follow any of the grouping schemes literally, but combined ideas from several schemes.

The clusters created by 27 participants were entered into IBM's EZSort analysis tool and a dendogram was produced (the dendogram formulated as a table is shown in Table 2). The names describing the categories were created by us to describe the groups.

The dendogram showed a division into two main categories, which we named "Multimedia" and "Memories". "Multimedia" contained three subcategories "Web", "TV and Video" and "Audio". "Memories" on the other hand also contained three subcategories, "Calendar", "Messaging" and "Photos and Personal videos".

"Web" subcategory included clearly all web related content that wasn't related directly to audio, video or images. All public/commercial video content was placed in "TV and Video", which contained both live TV and video streams as well as TV recordings and downloaded video clips. However, it did not contain any photos and videos taken by the user as those were placed under "Photos and Personal videos" in "Memories". In a similar fashion, videos and photos received from others were considered separate from public/commercial multimedia content and placed also to the same subcategory. "Audio" on the other hand contained all audio related items from personal music and podcasts collection to FM radio and online audio feeds.

"Calendar" subcategory in "Memories" included items related to calendar events, multimedia reminders and notes, which was quite similar content than in current calendar applications both in mobile devices and computers. "Messaging" was also following relatively clearly the messaging model existing in current mobile devices as it contained all messaging related items. The only subcategory in "Memories" containing non-personal information items was the mentioned "Photos and Personal videos", which users placed in their clusters closer to personal information items than other multimedia items.

We wanted also to compare the clusters created by Finnish and US participants to see, what kind of an effect the double amount of Finnish participants had to the all participants' dendogram. The biggest difference was found from the location of "Photos and personal videos" subcategory. Finnish participants had placed it similarly than in all participants' dendogram to "Memories" with one small difference as the received photos and videos which were placed to "Messaging" subcategory. US participants on the other hand had clearly separated photos from videos. All selfcreated, received and downloaded images were placed together, while self-created and received videos were kept separated from photos (both under "Multimedia"). 
Table 2. Dendogram created from all participants' clusters in detail

\begin{tabular}{|c|c|c|c|}
\hline Category & Subcategory & Folder & Content \\
\hline \multirow[t]{6}{*}{ Multimedia } & Web & Network folder & Network folder \\
\hline & & Web pages & $\begin{array}{ll}\text { Web notifier } & \text { (RSS), } \\
\text { URL, } & \text { Online } \\
\text { newspaper } & \end{array}$ \\
\hline & TV and Video & Collection & $\begin{array}{l}\text { TV recording, } \\
\text { Downloaded video clip }\end{array}$ \\
\hline & & Online & $\begin{array}{l}\text { TV channel, Video } \\
\text { stream }\end{array}$ \\
\hline & Audio & Online & $\begin{array}{l}\text { Radio channel, Audio } \\
\text { Stream }\end{array}$ \\
\hline & & Collection & $\begin{array}{l}\text { Audio book, Podcast, } \\
\text { Song, Ringtone, } \\
\text { Recorded music }\end{array}$ \\
\hline \multirow[t]{6}{*}{ Memories } & Calendar & Multimedia & Radio reminder, TV \\
\hline & & $\begin{array}{l}\text { Reminders } \\
\text { Calendar entries }\end{array}$ & $\begin{array}{l}\text { reminder } \\
\text { Calendar entry }\end{array}$ \\
\hline & & Notes & $\begin{array}{l}\text { Picture note, Note, } \\
\text { Audio note }\end{array}$ \\
\hline & Messaging & - & $\begin{array}{l}\text { Email, MMS, Text } \\
\text { Message }\end{array}$ \\
\hline & $\begin{array}{l}\text { Photos and } \\
\text { Personal videos }\end{array}$ & Received & $\begin{array}{l}\text { Received video, } \\
\text { Received image }\end{array}$ \\
\hline & & Albums & $\begin{array}{l}\text { Video shot with } \\
\text { camera, Downloaded } \\
\text { picture, Photo taken } \\
\text { with camera }\end{array}$ \\
\hline
\end{tabular}

\section{Background Study and Card Sorting Results Comparison}

Next, the dendogram created from all participants' clusters was compared to the information architecture models reviewed in the background study to see, how the information architecture models matched participants' mental models.

Similarly than in the background study results, the card sorting results quite clearly showed that the prevalent method was to categorize the different multimedia and personal information items according to personal information and media typology. Messaging and calendar items were included in own subcategories in both studies, and multimedia items had their own main and subcategories. The only difference to this was the inclusion of photos and personal videos closer to personal information items than public/commercial multimedia items as shown in the dendogram. This was drastically different compared to the reviewed information architecture models.

When comparing the personal information related subcategories, "Messaging" and "Calendar" were almost identical with the reviewed information architecture models. The only new items in "Calendar" were radio and TV reminders. Also notes were combined close to calendar items as in iPod, N80 and W800i currently.

With multimedia items there were more differences. In N80 and W800i, FM radio and music collection were clearly separated from each other. However, the dendogram 
showed clearly that participants saw closeness between them. Also, participants liked to have streamed audio close to other audio items.

In videos a clear difference could be seen. As stated in the background study results, none of the devices made any distinction between the self-created and public/commercial videos. However, the dendogram quite clearly showed that participants made this kind of distinction. And as an addition to that, participants also saw self-created photos and videos close together as already in N80, and place this new category closer to personal information items than multimedia items. With public/commercial videos there were similarities with music, as participants wanted both the local and the online content stored close to each others. Also, participants saw received items to be separated from self-created in "Photos and personal videos".

While doing the background study, we did not consider web content, such as web browser or web links, to be either multimedia or personal information and we left it out of the scope of the study. However, couple of web items was included to the card sorting study to see how participants would categorize them. The dendogram proposed web items to be included to "Multimedia", but this requires further research.

\section{Design Proposal}

Based on the background study and card sorting results we created a design proposal for optimal information architecture model in a mobile multimedia device as shown in Fig. 2. For the most part, it followed the dendogram.

The main categories of our design proposal were "Multimedia" and "Personal information". When compared to the dendogram, the biggest change was that "Photos and Personal videos" subcategory was moved to "Multimedia". We made this move, because participants' opinion was quite divided on this issue and we wanted to create a single entry point for all multimedia items. Other subcategories under "Multimedia" were "Audio", "TV and Video" and "Games".

As proposed by participants, both local and online items of the same multimedia type should be placed close together, but still clearly keep them separated from each other to different folders, namely "Collection" and "Online". The main reason for this was to clearly indicate, what content is always available and what might cause data traffic costs and delivery delays. Broadcasted content, such as Mobile TV and FM radio, was separated from other online content, because it usually has different pricing model and is only available in certain locations. Similarly audio and video podcasts were placed to own subcategories, because they contain both local and online aspects and for that reason do not fit to either subcategory. One more thing participants required was to separate your own content from received content. This was achieved by adding "Received items" folder to each collection/albums subcategory.

In "Audio", "Music collection" contained all local music items with typical artists, albums, songs, etc. categories, while "Online" had internet radio feeds and music shops. In "TV and Video" on the other hand, "Video collection" contained all local public/commercial video items, such as downloaded video clips and TV recordings, while "Online" had video streams and download services. In "Photos and Personal videos", "Albums" contained all photo albums including personal videos, while "Online" contained photo and personal video sharing services. 


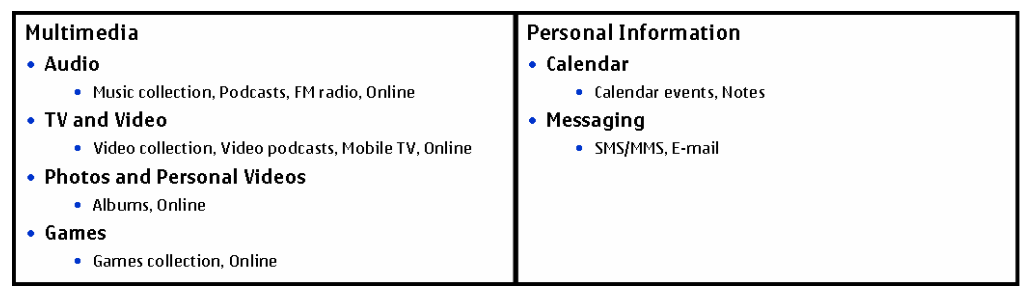

Fig. 2. Design proposal for an optimal information architecture in a mobile multimedia device

Games was one multimedia type we had left out from the card sorting cards, but we saw that games would fit quite naturally to "Multimedia" as they are already included to current devices, like PSP. As mentioned in the previous chapter, "Web" subcategory requires further research and was now left out of this design proposal.

"Personal information", on the other hand, had the remaining subcategories "Calendar" and "Messaging" of "Memories" category of the dendogram. Instead of only calendar events, "Calendar" subcategory would also contain notes as already in calendar applications in computer world, such as in MS Outlook. The correct place for multimedia reminders remains an open question and needs further research, because it is both related to multimedia items and calendar reminders. "Messaging" subcategory would contain all messaging related items and types as already in current mobile multimedia devices.

\section{Conclusions and Future Work}

In our research, we first reviewed information architecture models of four currently popular mobile multimedia devices. Then we used Open card sorting method to see what kind of mental models participants had for an optimal information architecture model in mobile multimedia devices. The comparison between the results showed:

- Photos and personal videos were placed closer to personal information items than public/commercial multimedia items

- There was a clear distinction between self-created and public/commercial videos

- Both local and online content of same multimedia type should be combined under same subcategory, but still keep a clear distinction between these subcategories

- Received items should be separated from your own, but still placed close

Based on the study results we created a design proposal for optimal information architecture model that followed the dendogram, but it also had some differences:

- "Photos and Personal videos" subcategory was placed under "Multimedia" instead of "Personal information", to create a single entry point for all multimedia items

- Local, online, broadcasted and podcast items of same multimedia type were placed close to each other, but still clearly separated

- Gaming items were missing from the card sorting study, but "Games" was added to "Multimedia", because games are already included to mobile multimedia devices 
- "Web" subcategory was not included to "Multimedia", because the opinion of participants was quite divided on this issue. The card sorting results proposed it to be included to "Multimedia", but this requires further research.

We believe that the proposed information architecture model would make mobile multimedia device UIs clearer, more usable and closer to the users' mental models. The design proposal is going to be validated with a further user test by using a Flash Lite prototype implementation of a mobile media player.

Acknowledgments. We would like to thank Gilles Baudet and Petri Piippo for their participation to this research project.

\section{References}

1. Dong, J., Martin, S., Waldo, P.: A User Input and Analysis Tool for Information Architecture. In: CHI '01: Extended Abstracts on Human Factors in Computing Systems, pp. 23-24. ACM Press, New York (2001)

2. Ewing, G., Freer, Y., Logie, R., Hunter, J., McIntosh, N., Rudkin, S., Ferguson, L.: Role and Experience Determine Decision Support Interface Requirements in a Neonatal Intensive Care Environment. J. of Biomedical Informatics 4/5, 240-249 (2003)

3. Henderson, S.: Genre, Task, Topic and Time: Facets of Personal Digital Document Management. In: CHINZ'05: Proceedings of the 6th ACM SIGCHI New Zealand Chapter's International Conference on Computer-Human Interaction, pp. 75-82. ACM Press, New York (2005)

4. Hudson, W.: Playing Your Cards Right: Getting the Most from Card Sorting for Navigation Design. interactions 5, 56-58 (2005)

5. Maiden, N.A.M., Hare, M.: Problem Domain Categories in Requirements Engineering. Int. J. Hum.-Comput. Stud. 3, 281-304 (1998)

6. Nielsen, J.: Usability Engineering. Morgan Kaufmann Publishers, Washington (1994)

7. Nielsen, J., Sano, D.: Sun Web: User Interface Design for Sun Microsystem's Internal Web. Comput. Netw. ISDN Syst. 1(2), 179-188 (1995)

8. Sinha, R., Boutelle, J.: Rapid Information Architecture Prototyping. In: DIS '04: Proceedings of the 2004 Conference on Designing Interactive Systems, pp. 349-352. ACM Press, New York (2004)

9. Upchurch, L., Rugg, G., Kitchenham, B.: Using Card Sorts to Elicit Web Page Quality Attributes. IEEE Softw. 4, 84-89 (2001)

10. Vredenburg, K., Mao, J.-Y., Smith, P.W., Carey, T.: A Survey of User-Centered Design Practice. In: CHI '02: Proceedings of the SIGCHI Conference on Human Factors in Computing Systems, pp. 471-478. ACM Press, New York (2002) 